\title{
Cultura de aprendizaje: factor relevante en la enseñanza por tareas del español
}

\author{
Alejandra Vela Hidalgo y Yudi Buitrago Rico \\ Pontificia Universidad Católica del Ecuador
}

(Texto recibido el 05 de octubre de 2018; aceptado el 16 de marzo de 2019; versión final el 18 de marzo de 2019)

DOI: https://doi.org/10.5565/rev/jt13.778

Resumen: Este artículo explora los factores de éxito o fracaso, específicamente la cultura de aprendizaje, de la enseñanza basada en tareas en clases de español como segunda lengua para promover el uso de la lengua meta fuera de clase. Para esto, se llevaron a cabo dos estudios de caso con dos grupos de diferentes niveles (A1-A2 y B2). Los resultados apuntan a que el uso de tareas fuera de clase ayuda a los estudiantes a usar la lengua meta en contextos auténticos, siempre y cuando el aprendiente esté familiarizado con dicha metodología y/o esté dispuesto a aceptarla. En el caso de estudiantes de culturas de aprendizaje diferentes, se encontraron dificultades que no afectaron el resultado final. Se concluye que el enfoque por tareas es una herramienta exitosa para fomentar el uso de la lengua meta fuera del aula cualquiera que sea la cultura de aprendizaje de los aprendientes.

Palabras clave: enseñanza por tareas, inmersión, español para asiáticos, cultura de aprendizaje

\begin{abstract}
In this study, we explore the key factors of Task Based Language Teaching in Spanish as L2 classes in immersion contexts, to encourage the use of the target language outside the classroom. In order to do this, we developed two case studies, with two groups of different proficiency levels (A1-A2 and B2). The results show that it is true that the use of Task Based Teaching outside the classroom setting helps students to use the target language in real contexts. However, this is only true when students are acquainted with the methodology and/or willing to learn and accept it. In some cases, where the learning culture of the students was different, we did find difficulties. We conclude that, despite the students' learning cultures, Task Based Language Teaching is indeed a useful and successful classroom tool to foster the use of the target language outside the classroom and in real contexts.
\end{abstract}

Keywords: task based teaching, immersion, Spanish for Asians, learning culture

Résumé : Cet article explore les facteurs de succès ou d'échec, spécifiquement liés à la culture d'apprentissage, de la méthodologie basée sur l'utilisation de tâches dans une classe d'espagnol comme seconde langue pour promouvoir l'usage de la langue cible en dehors du cours. Pour cela, deux études de cas ont été réalisé avec deux groupes de différents niveaux (A1-A2 et B2). Les résultats montrent que l'usage de projets en dehors de la classe incite les étudiants à utiliser la langue cible dans des contextes authentiques, à condition qu'ils soient familiarisés avec cette méthodologie et/ou disposés à l'accepter. Dans le cas d'étudiants issus de différentes culture d'apprentissage, des difficultés ont été observé sans que cela affecte le résultat. On 
finit par constater que l'enseignement basé sur des tâches et des projets se montre excellent pour promouvoir l'usage de la langue cible en dehors de la salle de classe, et ce, quel que soit la culture d'apprentissage des étudiants.

Mots-clés : enseignement par tâches, immersion, l'espagnol pour des asiatiques, une culture d'apprentissage

\section{Introducción}

En este artículo se sintetizan los resultados de un proceso investigativo, en el que se indaga sobre la enseñanza basada en tareas (EBT) en la clase de español como segunda lengua (L2) en contextos de inmersión. Nuestro punto de partida es analizar y comprender cómo las percepciones influenciadas por la cultura de aprendizaje de los estudiantes pueden contribuir a que la EBT sea exitosa en contextos de inmersión y con grupos heterogéneos. Este estudio nace del cuestionamiento sobre si la EBT es el enfoque más adecuado para promover el uso de la lengua fuera de la clase cuando las percepciones de los estudiantes sobre el tipo de actividades no son favorables.

La idea de este estudio surgió porque, en nuestra escuela de español en una universidad ecuatoriana, se notó que los aprendientes que venían de países orientales muchas veces priorizaban el aprendizaje de la gramática y asumían con resistencia el uso de actividades comunicativas y más aún si éstas implicaban salir de la clase. Además, se vio un hueco en la literatura disponible (Appel y Gilabert, 2002; Burrows, 2008; Córdova Zúñiga, 2016; Lai, Zhao, y Wang, 2011; Markovic y Lazarevic, 2010; McDonough y Chaikitmongkol, 2007; Sultana y Zaki, 2015), donde la ETB era siempre exitosa con grupos culturalmente homogéneos y no en contextos de inmersión. Por lo tanto, planteamos la necesidad de hacer una investigación sobre el uso de la ETB con grupos de diferentes orígenes en un contexto de inmersión y que evalúe cómo sus percepciones contribuían o perjudicaban los resultados.

\section{Marco teórico: cultura de aprendizaje}

Cuando se afirma que el enfoque por tareas es exitoso, se deben establecer ciertos parámetros para entender bajo qué condiciones lo es. Para muchos instructores y estudiantes, esto no es necesariamente verdad pues cada uno, con su respectiva cultura, tiene una idea de lo que debe ser o no una clase. "Although it is not always immediately apparent, everything we do in the classroom is underpinned by beliefs about the nature of language and about language learning" (Nunan, 1989, p.12). Por esto algunos estudiantes pueden tener reacciones que dificulten la puesta en marcha de ciertos enfoques con los que no están familiarizados. 
Se entiende como cultura de aprendizaje a las creencias que tanto estudiantes como profesores tienen sobre cómo se enseña y aprende. Éste es un factor que determina lo que sucede en la clase y si se lo ve como exitoso o no (Cortazzi y Jin, 1996). Cortazzi y Jin (1996, p. 169) define al concepto de "cultura de aprendizaje" como:

By the term 'culture of learning' we mean that much behaviour in language classrooms is set within taken-for-granted frameworks of expectations, attitudes, values and beliefs about what constitutes good learning, about how to teach or learn, whether and how to ask questions, what textbooks are for, and how language teaching relates to broader issues of the nature and purpose of education.

Muchas veces, las culturas de aprendizaje son algo invisible que tomamos como natural cuando en realidad estas creencias están supeditadas a una sociedad respectiva. Sánchez Griñán (2008, p. 403) explica que "Las raíces de una cultura de aprendizaje se encuentran en las tradiciones educativas y culturales de una determinada sociedad". Este concepto es importante porque en las clases heterogéneas, las culturas de aprendizaje suelen estar en constante interacción.

Así, por ejemplo, muchos estudiantes de la China vienen de sistemas tradicionales de aprendizaje de lenguas, herederos de los métodos basados en la traducción y el método audiolingual, que fueron introducidos en ese país con el afán de conocer los textos de la cultura europea. "La traducción de los textos de la civilización occidental desarrollada era el objetivo ineludible de la enseñanza de idiomas, que debía servir, ante todo, a la prosperidad del país" (Sánchez Griñán, 2008, p. 309). Lo que continuó hasta los ochenta pero que tiene todavía resonancias. Muchos autores han mencionado que el sistema de enseñanza de lenguas en China está basado hoy en día en:

la traducción y la adquisición mediante repetición y memorización... Existe lo que se denomina «cultura china de aprendizaje», en la que predomina la jerarquía junto con el pánico al error. En cuanto a la jerarquía hablamos de un profesor como director de escena y unos estudiantes supeditados a sus preceptos (Castellano Merino, Arjonilla Sampedro, y Sánchez López, 2011, p. 590)

El problema surge cuando estos estudiantes son introducidos en culturas de aprendizaje diferente que no entienden porque son ajenas a ellos. 


\section{Marco teórico: enseñanza basada en tareas}

La EBT ha tenido relevancia en las últimas décadas y ha permitido que el aprendizaje de lengua se produzca en contextos más auténticos, empoderando a los estudiantes, pero manteniendo la guía del instructor. Según Long y Crookes (1992), este enfoque se concentra en cómo se aprende y no en lo que se aprende. Para estos autores, no debe haber una preselección de temas, sino que estos deben ser negociados entre el instructor y los aprendientes en el transcurso de la clase, enfatizando así el proceso de aprendizaje y no los contenidos a ser aprendidos. Los aprendientes de una L2 necesitan oportunidades para recibir suficiente input (si es posible auténtico) y luego para participar en actividades comunicativas reales. En todos los casos, se intenta que este proceso sea significativo para el aprendiente.

Justamente en la EBT, el propósito de los estudiantes es completar una tarea a través del uso de la lengua meta. Nunan (1989, p. 10) define las tareas en el contexto de aprendizaje de lengua como:

a piece of classroom work which involves learners in comprehending, manipulating, producing or interacting in the target language while their attention is principally focused on meaning rather than form. The task should also have a sense of completeness, being able to stand alone as a communicative act in its own right.

Para Livingstone y Ferreira (2009, p. 91), la EBT es el método perfecto "para desarrollar y mejorar la competencia funcional en una lengua extranjera sin sacrificar la exactitud gramatical." En este sentido, este enfoque es adecuado para resolver un problema muy común y concreto: "los estudiantes no siempre muestran tener la competencia comunicativa para desenvolverse en la vida real fuera del aula. En efecto, su competencia en la lengua meta a veces es más lingüística que comunicativa" (Livingstone y Ferreira, 2009, p. 92). Muchos estudiantes de clases tradicionales son capaces de explicar las reglas gramaticales pero no de usarlas de manera espontánea. Al usar la EBT, se busca incorporar actividades que promuevan una comunicación auténtica dentro de cierto contexto de control. El objeto es que no desarrollen solamente su competencia lingüística sino también la comunicativa.

Para Estiarte y Zanon (1990, p. 58), la noción de competencia comunicativa está "en el centro de un «contínuum» delimitado en un extremo por una dimensión formal (el conocimiento sobre el lenguaje) y en el otro por una dimensión instrumental (la capacidad de usar el lenguaje)". Para afianzar esta competencia es necesario "el ejercicio de los procesos (conocimiento instrumental) que convierten esos contenidos en acciones de comunicación", 
características de la interacción natural del ser humano. En el aula, este tipo de ejercicios implica "el despliegue de estrategias de uso del instrumento lingüístico con el objetivo de solucionar problemas concretos (de fluidez, interpretación de significados, léxico, etc.), en relación a las tareas propuestas". Según estos mismos autores, las tareas, representando a la vida real o, en sistemas de inmersión, como parte de ella, contienen los aspectos lingüísticos, determinan los contenidos y sirven como evaluación.

En general, la EBT en el mundo académico de occidente es considerada como exitosa en el sentido que ofrece contextos auténticos de comunicación en los que los aprendientes tienen que usar la lengua como medio para cumplirlas (Nunan, 1991). La tarea es lo más cercano a lo que hacen los hablantes nativos: comunicar y lograr algo. Cuando la tarea sucede en la clase, el aprendizaje adquiere más autenticidad en la interacción aunque no necesariamente de la situación (Ellis, 2017), lo que no es necesariamente verdad en contextos de inmersión, donde las situaciones pueden ser totalmente auténticas.

\section{Marco teórico: EBT, aspectos problemáticos}

Sin embargo, se han señalado algunos problemas cuando el enfoque ha sido usado en contextos no occidentales. Burrows (2008, p. 11). advierte que: "This interaction, at the heart of TBL, means success (however one measures it) depends on the degree and nature of student involvement." Es decir, que la EBT funciona siempre y cuando los estudiantes tengan buenas actitudes. Agrega que las expectativas de aprendizaje independiente y autorregulado vienen de una cultura de aprendizaje occidental. Este autor afirma que la suposición de que a los estudiantes les guste trabajar independientemente del profesor no es necesariamente cierta, pues en culturas, como la japonesa, muchos aprecian las oportunidades de interactuar con él.

Hay que considerar que si bien el desarrollo de la EBT en lenguas ha tenido un gran avance en las últimas décadas en Europa y Estados Unidos, esto no es necesariamente real para todo el mundo. Pastor Gutiérrez $(2016$, p. 8) explica que:

La cultura de aprendizaje del alumno es la que determinará su visión de todo cuanto sucede en clase; su papel como alumno y compañero, el papel que espera que cumpla el profesor, su grado de participación, su idea de lo que constituye un buen método de enseñanza/aprendizaje, en qué debe consistir la evaluación, etcétera.

Por lo tanto, en una sola clase puede haber tantas culturas de aprendizaje como estudiantes, especialmente en contextos como el de este estudio, donde recibimos estudiantes de todas 
partes del mundo. Tomando en cuenta esto, es lógico que algunos estudiantes puedan mostrar resistencia cuando se les pide cumplir tareas en contextos reales.

En clases de lenguas con estudiantes chinos en el mundo occidental, algunos autores (Aristu Ollero, 2014; Pastor Gutiérrez, 2016; Blanco, 2013) afirman que les toma más tiempo aprender una lengua mediante enfoques comunicativos porque, antes de empezar a aprender, deben adaptarse al estilo de enseñanza y aprendizaje. No sólo tienen que aprender la lengua, sino también la cultura (dentro de la cual está el sistema educativo) (Aristu Ollero, 2014). Para Aristu Ollero (2014), hay que permitirles y darles tiempo de adaptarse. Poco a poco se van acostumbrando a las nuevas dinámicas. También hay estudios, como el de Pastor Gutiérrez (2016), que afirman que los estudiantes chinos aprecian la enseñanza comunicativa de idiomas.

Por otro lado, Sánchez Griñán (2008) asegura que la enseñanza de idiomas por tareas en China todavía tiene resistencia, no tanto porque los profesores o los alumnos no crean en sus beneficios, sino porque hay una presión por parte del gobierno de mantener un sistema tradicional al exigir a los estudiantes aprobar exámenes nacionales en los que se evalúa el aspecto gramatical. Esto cierra las opciones de implementar enfoques comunicativos.

\section{Metodología}

Nuestro objetivo es analizar cómo las percepciones sobre la EBT, influenciadas por las culturas de aprendizaje de los estudiantes de español como lengua extranjera en contextos de inmersión, influyen en los resultados de aprendizaje, específicamente en el uso de la lengua meta en contextos más auténticos. La hipótesis que se defiende es que la cultura de aprendizaje facilita o dificulta el proceso de la EBP en la clase de español (L2), pero siempre promueve el uso de la lengua meta en contextos auténticos. Se formularon las siguientes preguntas para el contexto de la enseñanza de español como L2 en un contexto de inmersión: ¿qué percepciones tienen los estudiantes de diferentes orígenes sobre la EBT? ¿Es la cultura de aprendizaje un factor que facilita o dificulta la implementación de la EBT?

La presente investigación tiene un enfoque cualitativo para lo cual se usaron dos estudios de caso para evaluar cómo las percepciones de los aprendientes afectaban o no la implementación de la EBT en el uso de la lengua fuera de la clase.

Para el análisis de datos, debemos aclarar que no se realizó un proceso lineal que inicie con una etapa de recolección de información y luego otra de análisis, sino que cada momento de recolección parcial se acompañó de su respectivo análisis, por un lado, para 
realizar un seguimiento y evaluación de los instrumentos, y por otro, para ir organizando el cuerpo de datos. Se trató entonces de ciclos de recolección y análisis.

A continuación se describe el proceso de estos ciclos: Cada información obtenida de las diversas fuentes fue capturada y organizada: las encuestas, las notas de las investigadoras y los proyectos se organizaron en un archivo para su sistematización. A continuación, se efectuó una codificación a partir de las categorías de análisis: cultura de aprendizaje, uso de la lengua en contextos auténticos y enfoque por tareas. El proceso de codificación fragmentó las transcripciones en categorías separadas de temas, conceptos, eventos o estados; y mostró cada detalle y cita textual, para determinar en qué aportaba al análisis. Una vez se hubo codificado, se procedió a relacionar la información para establecer cómo se relacionaban las categorías de análisis. En resumen, se buscó ir procesando los datos a medida que se los iba recolectando, generando un ir y venir, aplicando las estrategias analíticas, e incrementando el grado de validez y confiabilidad con una constante evaluación y retroalimentación.

\section{Recolección de datos}

El estudio se llevó a cabo en una universidad privada en Quito, Ecuador, en las clases de español para extranjeros en 2017, con dos grupos. Uno estaba cursando el nivel 2 del programa, que corresponde a la transición de A1 y A2 según el Marco común europeo de referencia (MCER). El otro fue el nivel 6, que corresponde al B2 según el MCER. Ambos cursos están diseñados como cursos intensivos en contexto de inmersión durante ocho semanas, tres horas diarias de clase de lunes a viernes, con un total de 120 horas. Los estudiantes que participaron en el estudio pertenecen a distintas nacionalidades y contextos lingüísticos; además, su estancia en Ecuador variaba, así como sus niveles de educación. En este sentido, el grupo se caracterizó por la heterogeneidad. La mayoría eran adultos jóvenes que tenía al momento del estudio entre 17 y 38 años, excepto por una estudiante de Corea que tenía 49 años. En el Anexo 1 se puede apreciar y comparar la información de los participantes.

Se recolectaron tres tipos de datos: las notas de campo de las profesoras que impartieron el curso, los proyectos finales de los estudiantes y las encuestas de evaluación final, en las que los estudiantes tuvieron la oportunidad de expresar lo que pensaban sobre el enfoque y su propio desempeño. Primero, las notas de campo de las profesoras constituyen las anotaciones semanales que hicieron durante las ocho semanas de curso. Segundo, los proyectos son el resultado de una serie de tareas que iban cumpliendo a lo largo de las dos 
semanas (ver ejemplo en Anexo 2). Finalmente, la encuesta (Anexo 3) indaga por las actividades realizadas en clase y la valoración dada a cada una, en función del grado de utilidad que los estudiantes les asignaron. La idea fue poder contrastar las percepciones de los estudiantes sobre los diferentes tipos de tareas. Aunque el objetivo fue analizar las opiniones sobre los proyectos, se incluyeron otros tipos de actividades para que los participantes no supieran el propósito, y poder contrastar la información. En detalle, las actividades sobre las que se les preguntó fueron: Escrituras (textos como cartas, pequeños relatos, correos electrónicos, ensayos, etc.); Talleres de conversación (interacción oral a partir de diversos temas); Proyectos (Tareas con fines de proyecto: actividades investigativas que se van acumulando y que sumadas construyen un proyecto); y Pruebas (evaluaciones de todas las competencias).

\section{Acciones didácticas: nivel A1-A2}

Los proyectos que entregaron los estudiantes fueron: un folleto sobre un evento cultural y un mapa de locales comerciales, en los cuales, los aprendientes tuvieron la libertad de escoger el tema. Por ejemplo, dentro de los eventos, seleccionaron conciertos, exposiciones de arte, desfiles, fiestas tradicionales, etc. Para completar estos proyectos, los estudiantes tuvieron que seguir una guía proporcionada por la profesora, la cual consistía en actividades paso por paso para llegar al producto final (Anexo 4). Para el mapa de locales comerciales, el objetivo fue que los alumnos practicaran el pedir y dar información sobre lugares de la ciudad. Primero, los aprendientes tuvieron que determinar el tipo de comercios: peluquerías, cafeterías, restaurantes, etc. Luego, investigar en internet sobre los locales que existían en la urbe y planificar visitas. Ya en los lugares, tuvieron que hacer preguntas a los dependientes sobre precios y productos. Finalmente, con la información recolectada, crearon un mapa de la ciudad donde ubicaron los sitios y escribieron la información más relevante, que compartieron con sus compañeros.

\section{Acciones didácticas: nivel B2}

En el nivel B2, el proceso didáctico se desarrolló de la siguiente manera. En la primera semana de clase, cada estudiante escogió un tema que le resultara atractivo (a partir de sus intereses y expectativas). Luego se hizo una lluvia de ideas de cada tema y cada estudiante planteó la pregunta guía (por ejemplo, ¿cómo desarrollarías un plan de negocios para exportar palmito ecuatoriano?, ¿qué podrías hacer para ayudar a las madres extranjeras residentes en 
Quito y cuyas familias viven lejos?, ¿qué campaña realizarías para evitar la piratería de libros? ¿por qué hay tantos perros callejeros en Quito?,etc.). En la siguiente semana, debían buscar información sobre ese tema en relación específicamente a Ecuador o Latinoamérica. Esto lo hicieron de manera libre en internet, y trajeron a clase sus impresiones o nuevas preguntas. Este punto del proceso fue muy provechoso porque encontraron diversos formatos, registros y debates, lo cual enriqueció su acervo léxico y discursivo. La tercera semana, debían plantear el producto que desarrollarían como resultado final del proceso; así, dos estudiantes presentaron campañas preventivas, otros dos, planes de negocios, y otro, un plan para la escritura de un libro. La cuarta semana se focalizó en la búsqueda de información, por lo cual, se les pidió que hicieran una entrevista a alguien (nativo en lengua castellana) que fuera conocedor del tema, y que buscaran información únicamente en páginas web de instituciones reconocidas que trabajaran en el ámbito seleccionado. Durante la quinta y sexta semanas, mostraron informes parciales de sus avances. Finalmente, en la séptima semana presentaron el producto final. Para terminar, se hizo una reflexión sobre el enfoque de aprendizaje por tareas, y cada uno compartió una autoevaluación y evaluación.

\section{Resumen de los resultados de las acciones didácticas}

En la encuesta final, se preguntó a los aprendientes de nivel A1-A2 acerca de cuál de todas las actividades que desarrollaron durante el curso pensaban que les había ayudado a mejorar su español (Escritura, Taller de conversación, Tareas [actividades gramaticales], Proyectos, Pruebas). Aunque el objetivo era evaluar las percepciones de los estudiantes sobre los proyectos, vimos necesario tener datos con los cuales contrastar. Los resultados mostraron que tres estudiantes de China, la estudiante de Corea y la de Taiwán, estudiantes de culturas de aprendizaje más tradicional, marcaron en primer lugar las tareas (actividades gramaticales). En cuanto a los proyectos, solo dos personas los seleccionaron entre las actividades más útiles: la estudiante coreana y la ucraniana.

Cuando se les preguntó por las razones de su elección, los que escogieron las Tareas y Escritura explicaron que preferían estas actividades porque obtenía retroalimentación y eso le ayudaba a identificar falencias. Lo que está en coherencia con la observación de que en la EBT no es posible dar retroalimentación constante. Luego se les preguntó qué había sido lo más difícil al realizar los proyectos. A esta pregunta, la mitad respondió que la comunicación con personas que no conocían y entre compañeros en el contexto fuera de clase. Tres de los cuatro chinos afirmaron que la mayor barrera fue la falta de vocabulario para comunicarse y 
conocimiento de la cultura, lo que muestra que la categoría de cultura de aprendizaje es un factor determinante. La última pregunta se refería a cómo los proyectos les habían ayudado en su proceso de adquisición la lengua. Las respuestas reflejaron que la impresión de los aprendientes era que los proyectos les ayudaron para: aprender sobre la cultura latinoamericana y ecuatoriana; hablar con nativo hablantes; hablar sin mirar el libro o celular; escuchar la pronunciación correcta; y escribir independientemente. Es decir que, a pesar de que las impresiones de los aprendientes no siempre fueron positivas, los objetivos de los proyectos se cumplieron: la interacción en contextos auténticos.

En cuanto a las notas de campo de la profesora, se notó que había una reacción diferente hacia los proyectos entre los estudiantes de los países asiáticos y los de los más occidentalizados. Los estudiantes asiáticos (tres chinos y la coreana) mostraron algunas dificultades como la comunicación con sus compañeros y resistencia a ir a lugares que no conocían, como también lo señalaba la encuesta: "para mí difícil cuando mi compañero no entiende". Aunque cabe señalar esto no fue verdad para todos los estudiantes asiáticos pues la estudiante de Taiwán y la cuarta estudiante china tuvieron actitudes positivas hacia los proyectos.

En el análisis de los proyectos finales y en las notas de la profesora, se encontró que hubo desigualdad en la cantidad de trabajo que hicieron los aprendientes dentro de los grupos. Cuando el nivel de proficiencia era notorio, el estudiante con más proficiencia se benefició más del proceso. Al final del curso, hubo cierta resistencia de las estudiantes de Brasil y de Francia a trabajar con sus compañeros asiáticos porque "no sentían que aprendían al trabajar con ellos y era muy difícil la comunicación" (fuente: notas de la profesora). El estudiante de China explicó que para él era muy complicado hablar con sus compañeros de otros países y que prefería trabajar con gente de China. Por tanto, la cultura de aprendizaje fue un aspecto determinante.

La mayoría de proyectos resultó muy creativa y se vio que los estudiantes usaron la lengua satisfactoriamente en el proceso. Los aprendientes realizaron investigación independiente en español tanto en internet como a través de visitas, entrevistas en español y observación de comportamientos en lugares públicos. Por ejemplo, acudieron a eventos culturales en los que tuvieron que interactuar con otros asistentes, visitaron locales comerciales y averiguaron sobre los productos, probaron comida nueva, etc. En resumen, la EBT se mostró efectiva para usar la lengua meta en contextos reales: pudieron interactuar con hablantes nativos, aprender sobre la cultura local y usar la lengua meta para comunicarse. 
En el grupo B2, solo el estudiante de Corea señaló que las tareas de tipo gramatical fueron las más provechosas en su aprendizaje, mientras que el estudiante norteamericano seleccionó los talleres de conversación. Las tres estudiantes restantes (china, húngara e israelí) señalaron los proyectos como la actividad más útil ${ }^{1}$. Sobre las razones de su elección, el estudiante inclinado por las actividades gramaticales afirmó que "me sirven para mecanizar las estructuras". En cuanto al estudiante que prefería las actividades conversacionales, argumentó que "la pronunciación es mi punto más débil y debo practicar en ese ámbito". Los estudiantes que prefirieron los proyectos argumentaron que este enfoque les permitió aprender vocabulario informal o técnico y además se sentían avanzando en un sueño personal.

Sobre la dificultad para llevar a cabo los proyectos, los estudiantes señalaron que les faltó tiempo para hacer un mejor proyecto, puesto que algunos ya estaban trabajando, y era un tipo de ejercicio que demandaba mucho tiempo. Por otro lado, afirmaron que la interacción con personas nativas podía ser difícil por la falta de vocabulario y las dificultades en la comprensión, esto debido a que tres de ellos entrevistaron a nativos no ecuatorianos (español, argentino y chileno). Sobre lo que más les habían aportado las tareas para su aprendizaje, la mayoría argumentó que aprendieron sobre la cultura ecuatoriana, mejoraron su vocabulario, y conocieron sobre trámites y burocracia en Ecuador.

Como se aprecia en el diario de campo de en este nivel, se podría señalar que en general todos los estudiantes se sentían entusiasmados con el proyecto, a excepción de los dos coreanos, que mostraban desinterés e incluso hostilidad. La estudiante china estaba bastante comprometida con su proyecto, así que, dentro del grupo de asiáticos, fue la única que siempre mostró interés. Esto también se reflejó en el producto final, pues ella presentó un proyecto muy elaborado y creativo, mientras que el estudiante coreano presentó un proyecto menos prolijo y algo improvisado. En este caso, los dos tenían en mismo nivel de proficiencia lingüística, pero el estudiante coreano estaba más interesado en cuestiones gramaticales, y hacía mucho énfasis en esto. Incluso expresó que los proyectos eran una pérdida de tiempo. Los otros tres estudiantes fueron muy receptivos sobre los proyectos y cumplieron la tarea a cabalidad. Estos resultados muestran que en niveles más avanzados, los estudiantes muestran mejores opiniones sobre el enfoque por tareas y pueden comprender sus beneficios a través de las evaluaciones y guía del instructor. Sin embargo, es interesante, que un estudiante coreano insista en la preferencia por las actividades gramaticales.

Sobre los productos finales, cabe resaltar que, aunque algunos estudiantes lo asumieron con más seriedad que otros, en general, todos pudieron interactuar en diversas situaciones donde debieron enfrentarse a usar la lengua con hablantes nativos, y pudieron 
beneficiarse de esta interacción al aprender nuevo vocabulario y conocer más sobre las costumbres y la cultura locales. Así, a modo de ilustración, uno de los proyectos fue una campaña para la esterilización de mascotas en la ciudad de Quito, para ello, la estudiante indagó en diversas instituciones relacionadas con el bienestar animal; además, entrevistó a funcionarios de la entidad gubernamental encargada de actividades relacionadas con la fauna urbana, y finalmente, planteó una estrategia mediática en redes sociales para impulsar la esterilización de mascotas.

\section{Discusión de los resultados}

A continuación, se comentarán los resultados de los estudios de caso, para encontrar los factores que contribuyeron a un uso satisfactorio o no de la EBT en cuanto a la interacción en situaciones auténticas. Específicamente, se verá cómo la cultura de aprendizaje, nuestra categoría de análisis, es un factor que puede causar dificultades pero no es en realidad un impedimento para que el enfoque promueva el uso de la lengua en contextos reales en clases de español como lengua extranjera en inmersión.

Tanto en las encuestas como en las observaciones de las profesoras, se evidenció que en ambos niveles los aprendientes efectivamente usaron la lengua en contextos reales y rompieron los límites del aula. La profesora del nivel A1-A2 anotó: "A pesar de que el estudiante $\mathrm{X}$, de China, había mostrado preocupación con respecto a la idea de acudir a un evento cultural, ha ido a una exposición de arte con la estudiante Y (ucraniana), donde ha hablado con un nativo". Lo que se complementa con el comentario en la encuesta del mismo estudiante sobre su experiencia con los proyectos, donde dice: "Puedo hablar y no miro el teléfono", se refería al traductor del teléfono. En general, los aprendientes manifestaron que tuvieron la oportunidad de hablar con nativo hablantes. Ya sea como dificultad o como algo positivo, la mayoría expresó en la encuesta que tuvo que interactuar en instancias auténticas, y al hacer esto, aprendió vocabulario, se fijó en la entonación y/o conoció más sobre la cultura local.

Las respuestas a la primera pregunta de la encuesta están en concordancia con la influencia de la cultura de aprendizaje, porque efectivamente $80 \%$ de los aprendientes de origen asiático dio prioridad a las actividades gramaticales. Una excepción fue la estudiante china de nivel B2, quien desdeñaba de los ejercicios gramaticales y mostró entusiasmo en su proyecto. Esto se explica porque pasó sus estudios universitarios en Alemania, lo que le permitió modificar su cultura de aprendizaje. 
La estudiante francesa y la brasileña del nivel A2, y la israelí y la húngara del nivel B2, tomaron los proyectos como algo natural que les incentivaba a explorar diversos contextos, como muestran sus encuestas y las notas de las profesoras. La francesa comentó en la encuesta que hacer el proyecto de los locales comerciales (lo hizo sobre restaurantes vegetarianos) le había permitido conocer más la ciudad e incluso saber dónde podría solicitar un trabajo en el futuro. Por lo tanto, sus expectativas sobre lo que es aprender una lengua estaban en concordancia con el enfoque usado. En cuanto a los que mostraron dificultad y resistencia, como los coreanos de nivel B2 y los chinos del A1-A2, sus percepciones sobre la EBT les causaron dificultades.

Para entender por qué con estudiantes de culturas de aprendizaje más occidentales el enfoque funciona bien, comparamos dos casos extremos del nivel A1-A2: la estudiante francesa y un estudiante chino que mostró mucha dificultad en el proceso. Estas diferencias parecen estar relacionadas a dos aspectos: la cultura de aprendizaje y la lengua materna. A pesar de que la estudiante francesa tenía mucho menos tiempo residiendo en Ecuador al momento del estudio, obtuvo un nivel de desempeño superior. Creemos que esto es por su perfil: lengua materna romance, conocimiento de otras lenguas, familiarización con el sistema de aprendizaje y contacto diario con nativo hablantes. En contraste, el estudiante chino tenía otras características que no le favorecieron: lengua materna de origen sinotibetano, no había aprendido otras lenguas en el pasado, desconocimiento de la cultura de aprendizaje, poco o nulo contacto con hablantes nativos. El estudiante chino está claramente en desventaja para desempeñarse dentro del enfoque por tareas.

\section{Conclusiones}

Estos resultados preliminares apuntan a que la EBT del español como L2 en ámbito de inmersión es un sistema efectivo para que los estudiantes usen la lengua meta en contextos auténticos. Los participantes de ambos niveles interactuaron con hablantes nativos y se cuestionaron los límites de la clase como lugar de aprendizaje. Si bien algunos estudiantes provenientes de culturas de aprendizaje distintas no comprendieron el porqué de las actividades entorno a los proyectos, por la incompatibilidad de su cultura de aprendizaje, al final reconocieron que fue una forma adecuada para hablar en español con hablantes nativos. En contraste, los estudiantes con culturas de aprendizaje occidentales (independientemente de la nacionalidad) pudieron aprovechar eficientemente este enfoque. De manera general, podemos señalar que fue de utilidad para todos los estudiantes que, a pesar de vivir en un 
contexto de inmersión, no tienen la oportunidad de interactuar con ecuatorianos, como es el caso de muchos estudiantes chinos que trabajan y viven con gente de su propio país.

Se concluye además que un factor predominante en determinar el éxito o fracaso de la EBT en contextos de inmersión con grupos de estudiantes heterogéneos, son las percepciones que ellos tienen sobre el enfoque, las cuales dependen de sus culturas de aprendizaje formadas por experiencias pasadas.

Por lo tanto, se puede decir que la cultura de aprendizaje influencia cómo los estudiantes perciben el enfoque, aunque no necesariamente interfiere en el objetivo: interacción auténtica. Sin embargo, es evidente que este enfoque funciona mejor con estudiantes de culturas de aprendizaje más occidentalizadas. Lo que confirma la relación de dependencia de las categorías: cultura de aprendizaje y enfoque basado en tareas. Mientras un estudiante esté más familiarizado con el enfoque, por su experiencia pasada con la EBT, más facilidad tendrá de desenvolverse.

\section{Referencias}

Appel, C. y Gilabert, R. (2002). Motivation and task performance in a task-based web-based tandem project. ReCALL 14 (1), 16-31. DOI: https://doi.org/10.1017/S0958344002000319

Aristu Ollero, A. (2014). Dificultades y retos de la enseñanza de ELE a estudiantes chinos: una experiencia en una escuela de idiomas de Barcelona. Monográficos SinoELE 10, $58-71$.

Blanco, J. (2013). Escollos lingüísticos de los principiantes chinos de español como lengua extranjera: Causas y sugerencias pedagógicas. Hispania 96 (1), 97-109.

DOI: https://doi.org/10.1353/hpn.2013.0026.

Burrows, C. (2008). An evaluation of task-based learning (TBL) in the Japanese classroom. English Today 24 (4), 11-16. DOI: https://doi.org/10.1017/S0266078408000345

Castellano Merino, A., Arjonilla Sampedro, A., y Sánchez López, I. (2011) Recursos de español para sinohablantes. En C. Hernández González, A. Carrasco Santana, y E. Álvarez Ramos (Eds.), La red y sus aplicaciones en la enseñanza-aprendizaje del español como lengua extranjera (pp. 589-600). Valladolid: Asociación para la enseñanza del español como lengua extranjera. 
Córdova Zúñiga, E. (2016). Implementing task-based language teaching to integrate language skills in an EFL program at a Colombian university. Profile Issues in Teachers' Professional Development 18 (2), 13-27. DOI: https://doi.org/10.15446/profile.v18n2.49754

Cortazzi, M. y Jin, L. (1996). Cultures of learning: Language classrooms in China. En H. Coleman (Ed.), Society and the language classroom (pp.169-206). Cambridge: CUP.

Ellis, R. (2017). Position paper: Moving task-based language teaching forward. Language Teaching 50 (4), 507-526. DOI: http://doi.org/10.1017/S0261444817000179

Estiarte, S. y Zanón, J. (1990). El diseño de unidades didácticas en L2 mediante tareas: Principios y desarrollo. Comunicación, Lenguaje y Educación 7 (8), 54-90. DOI: https://doi.org/10.1080/02147033.1990.10820942

Lai, C., Zhao, Y., y Wang, J. (2011). Task-based language teaching in online ab initio foreign language classrooms. The Modern Language Journal 95 (Supplementary Issue: Research in and Around the Language Classroom: Qualitative and Quantitative Approaches), 81-103. DOI: https://doi.org/10.1111/j.1540-4781.2011.01271.x

Livingstone, K. A. y Ferreira, A. (2009). La efectividad de un modelo metodológico mixto para la enseñanza-aprendizaje de español como lengua extranjera. Boletín de Filología 44 (2), 89-118. DOI: https://doi.org/10.4067/s0718-93032009000100004

Long, M. y Crookes, G. (1992). Three approaches to task-based syllabus design. TESOL Quarterly 26 (1), 27-56. DOI: https://doi.org/10.2307/3587368.

Markovic, L. y Lazarevic, N. (2010). As they wrote it - Students' projects. Elope 7 (1), 89104. DOI: https://doi.org/10.4312/elope.7.1.89-104.

McDonough, K. y Chaikitmongkol, W. (2007). Teachers' and learners' reactions to a taskbased EFL course in Thailand. TESOL Quarterly 41 (1), 107-132. DOI: https://doi.org/10.1002/j.1545-7249.2007.tb00042.x.

Nunan, D. (1989). Designing tasks for the communicative classroom. Cambridge: CUP.

Nunan, D. (1991). Communicative tasks and the language curriculum. TESOL Quarterly 25 (2), 279-295. DOI: https://doi.org/10.2307/3587464. 
Pastor Gutiérrez, I. (2016). Actitudes de los alumnos chinos respecto al aprendizaje del español en España. Revista de Enseñanza de Español a Sinohablantes 14, 1-27.

Sánchez Griñán, A. (2008). Enseñanza y aprendizaje del español como lengua extranjera en China. Retos y posibilidades del enfoque comunicativo (Tesis doctoral). Universidad de Murcia.

Sultana, M. y Zaki, S. (2015). Proposing project based learning as an alternative to traditional ELT pedagogy at public colleges in Pakistan. International Journal for Lesson and Learning Studies 4(2) 155-173. DOI: https://doi.org/10.1108/ijlls-09-2013-0049.

\section{Notas}

${ }^{1}$ Desafortunadamente, uno de los estudiantes coreanos abandonó por razones personales el curso faltando tres semanas para terminar, así que no pudo presentar el proyecto, ni responder la encuesta.

\section{Anexos}

Anexo 1: Características de los participantes

\begin{tabular}{|l|l|l|l|l|l|l|}
\hline & Nacionalidad & Género & Edad & $\begin{array}{l}\text { Estancia en un } \\
\text { país hispano }\end{array}$ & Ocupación & Nivel \\
\hline 1 & Brasileña & F & 22 & 7 meses & $\begin{array}{l}\text { Estudiante } \\
\text { universitaria }\end{array}$ & A2 \\
\hline 2 & Francesa & F & 24 & 3 meses & Ambientalista & A2 \\
\hline 3 & Ucraniana & F & 22 & 2 meses & Lingüista & A2 \\
\hline 4 & Estadounidense & F & 27 & 2 meses & $\begin{array}{l}\text { Ama de casa / } \\
\text { profesional }\end{array}$ & A1 \\
\hline 5 & Egipcia & M & 26 & 4 meses & Traductor & A1 \\
\hline 6 & Egipcia & M & 25 & 5 meses & Traductor & A1 \\
\hline 7 & Taiwanesa & F & 29 & 5 meses & $\begin{array}{l}\text { Estudiante } / \\
\text { profesional }\end{array}$ & A2 \\
\hline 8 & China & F & 23 & 4 meses & $\begin{array}{l}\text { Estudiante / } \\
\text { profesional }\end{array}$ & A1 \\
\hline 9 & China & F & 22 & 4 meses & Mesera & A1 \\
\hline 10 & China & F & 18 & 1 año & Estudiante & A1 \\
\hline
\end{tabular}




\begin{tabular}{|l|l|l|l|l|l|l|}
\hline 11 & China & $\mathrm{M}$ & 18 & 8 meses & Estudiante & A1 \\
\hline 12 & Iraní & $\mathrm{F}$ & 18 & 9 meses & Estudiante & A1 \\
\hline 13 & Coreana & $\mathrm{F}$ & 49 & 5 meses & Ama de casa & B2 \\
\hline 14 & China & $\mathrm{F}$ & 28 & un año & Ama de casa & B2 \\
\hline 15 & Israelí & $\mathrm{F}$ & 27 & 8 meses & $\begin{array}{l}\text { Estudiante/ } \\
\text { profesional }\end{array}$ & B2 \\
\hline 16 & Húngara & F & 25 & 4 meses & $\begin{array}{l}\text { Estudiante/ } \\
\text { profesional }\end{array}$ & B2 \\
\hline 17 & Coreana & M & 32 & 1 año & $\begin{array}{l}\text { Estudiante/ } \\
\text { profesional }\end{array}$ & B2 \\
\hline 18 & Coreana & M & 26 & 2 años & $\begin{array}{l}\text { Estudiante/ } \\
\text { profesional }\end{array}$ & B2 \\
\hline 19 & Estadounidense & M & 38 & 2 años & $\begin{array}{l}\text { Estudiante/ } \\
\text { profesional }\end{array}$ & B2 \\
\hline
\end{tabular}

\section{Anexo 2: Ejemplo de guía utilizada para el proyecto en nivel A1-A2. Ruta}

\section{de locales comerciales}

Descripción:

En parejas, los estudiantes van a diseñar una ruta/mapa de locales comerciales. Por ejemplo:

restaurantes coreanos en Quito; las mejores peluquerías del norte de Quito.

Objetivos:

Investigar sobre un tema de interés.

Buscar información en internet.

Hacer entrevistas.

Crear una ruta/mapa de los locales comerciales interesante.

Hacer una presentación.

Pasos:

Decidan sobre qué tipo de locales comerciales pueden hacer la investigación: bares;

restaurantes, panaderías, supermercados, etc. Tienen que ser específicos. Por ejemplo, restaurantes de comida italiana.

Pregunta a una o unas personas que viven en Quito sobre los locales que conoce y qué saben. Busca en internet más información. 
Necesitas encontrar sobre 2 locales: dirección, teléfono, horarios, tres productos o servicios, alguna característica especial. Para esto puedes visitar el local, buscar en internet o llamar por teléfono.

En una hoja limpia y suelta, organiza la información en formato de mapa.

Prepara una pequeña presentación para la clase.

\section{Anexo 3: Encuesta final de curso}

Nacionalidad:

Edad:

Sexo:

Ocupación:

Tiempo de estancia en Ecuador:

De todas las actividades que hicimos en clase, marca con números las que más te ayudaron. El 1 es la actividad que más te ayudó y 5 la que menos te ayudó.
a. Escrituras
b. Talleres de conversación
c. Actividades gramaticales (tareas)
d. Proyectos
e. Pruebas semanales

Explica por qué la actividad número 1 para ti fue la que más te ayudó.

De los cinco tipos de actividades en la pregunta 1, di cuál te ayudó en las siguientes áreas:

f. Para escribir mejor en español

g. Para hablar español en situaciones reales

h. Para hablar mejor con tus compañeros

i. Para conocer la cultura ecuatoriana y latinoamericana

j. Para practicar y automatizar la gramática y el vocabulario

k. Para mejorar tu comprensión auditiva en español 
1. Para aprender de manera independiente

\section{¿Qué fue lo más difícil al hacer los proyectos?}

\section{Menciona algo que aprendiste haciendo los proyectos relacionado a tu aprendizaje de español.}

\section{Información de las autoras}

Alejandra Vela Hidalgo es Candidata a PhD en Literatura Latinoamericana, Máster en Lingüística del Español y en Literatura Latinoamericana de la Universidad de Purdue. Docente de español en la Pontificia Universidad Católica del Ecuador.

Email: avelah@puce.edu.ec

Yudi Buitrago Rico es Doctora en Ciencias Políticas en FLACSO, Ecuador, Máster en Política social, Pontificia Universidad Javeriana y en Lingüística del español, Instituto Carvo y Cuervo, Colombia. Docente de español en la Pontificia Universidad Católica del Ecuador.

Email: ybuitrago266@puce.edu.ec

Para citar este artículo:

Vela Hidalgo, A. y Buitrago Rico, Y. (2019). Cultura de aprendizaje: factor relevante en la enseñanza por tareas del español. Bellaterra Journal of Teaching \& Learning Language \& Literature, 12(1), 25-43. DOI: https://doi.org/10.5565/rev/jt13.778 